\title{
The Intrinsic Field of Photons and Applications
}

\author{
Andre Vatarescu \\ Fibre-Optic Transmission of Canberra, 32 Batman Street, Canberra, Australia \\ Authore-mail address:andre_vatarescu@yahoo.com.au
}

\begin{abstract}
Any photon, regardless of its origin, carries the same optical field profile. This feature and the quantum Rayleigh emissions generate temporally discrete groups of several photons in a resonant cavity incorporating one single quantum dot. A periodic stream of single photons is distorted by high-finesse optical cavities and the quantum Rayleigh spontaneous emission in a beam splitter. Groups of photons emerge from interferometric filters because of multiple internal reflections.
\end{abstract}

Key words: photonic optical field, pure and mixed quantum states, quantum Rayleigh scattering.

\section{Introduction}

The conventional interpretation of quantum optic experimental outcomes is based on global and mixed quantum states of one photon per radiation mode [1-2]. The corresponding wavefunctions are associated with the statistical distribution of a large ensemble of measurements conducted under identical conditions. "A single-photon source coupling only to a single loss channel would emit a state given by $|\Psi\rangle=\sqrt{\mathrm{P}_{0}}|0\rangle+\sqrt{\mathrm{P}_{1}}|\Psi(1)\rangle$ with $\mathrm{P}_{0}+\mathrm{P}_{1}=1$. $\mathrm{P}_{1}$ is the probability that a single-photon emission has happened..." [1], and $\left|\Psi^{(1)}\right\rangle=\int d \omega \psi(\omega) \hat{a}^{\dagger}(\omega)|0\rangle$ would be a superposition of Fourier spectral components $\psi(\omega)$ measured at the output [1], but each measurement identifies only one spectral component at any given time. According to this picture, the intrinsic optical field of a photon inside an active cavity would be described by a global distribution whose measurement builds up as the ensemble of detected photons grows.

The expectation value of the photonic optical field operator $\hat{a}$ would be proportional to $\left\langle\Psi^{(1)}|\hat{a}| \Psi^{(1)}\right\rangle=\sqrt{\mathrm{P}_{0} \mathrm{P}_{1}} \int \psi(\omega) d \omega$ implying that the zero-photon state contributes to each of the single measurements, although, at the level of one single and individual measurement, the photodetections of zero-photon and one-photon events are mutually exclusive, being the result of a photon-dipole interaction or lack thereof. Similarly, each photon is monochromatic, and space- and time-limited, and cannot be described by a spectral Fourier component which is constant in time and space. The appropriate representation would be based on the mixed timefrequency analysis which is available from the subject of Signal Analysis [3].

It is the notation of global wavefunctions with no spatial or temporal dependence that conjures up an effect of quantum nonlocality but the time- and space-dependent pure states contradict such an interpretation while providing physically meaningful interpretations for the experimental outcomes. A major shortcoming of the conventional theory [1-2] is the omission of the quantum Rayleigh spontaneous emission in a dielectric medium [4-5], which prevents a single photon from propagating in a straight line. Equally, the stimulated quantum Rayleigh emission enables a group of monochromatic photons to follow a straight line of propagation by having the other group photons recapture the absorbed photon, thereby preventing spontaneous emission. The quantum Rayleigh stimulated emission may also couple two counter propagating photons onto each other depending on their relative phase when reaching the same electric dipole [6-8].

The core element of these ensemble measurements is a symmetric beam splitter (BS) which provides two alternative pathways - A and B - for one single photon of number state $|1\rangle$ entering a BS, to reach a photodetector. When the two branches are brought jointly to the same detecting area, the emerging entangled state describing an ensemble distribution [9] would be $|\Psi\rangle_{\text {out }}=$ 
$\left(|1\rangle_{\mathrm{A}}|0\rangle_{\mathrm{B}}+|0\rangle_{\mathrm{A}}|1\rangle_{\mathrm{B}}\right) / \sqrt{2}$ giving rise to a mathematical non-vanishing interference term, in the form of $\left\langle\hat{a}_{\mathrm{A}}^{\dagger} \hat{a}_{\mathrm{B}}\right\rangle=\operatorname{Tr}\left(\rho \hat{a}_{\mathrm{A}}^{\dagger} \hat{a}_{\mathrm{B}}\right) \neq 0$, where the density matrix is $\rho=|\Psi\rangle_{\text {out out }}\langle\Psi|$, and the photon creation and annihilation operators are, respectively, $\hat{a}_{\mathrm{A}}^{\dagger}$ and $\hat{a}_{\mathrm{B}}$ of the two propagating modes. "The observed intensity pattern results from the interference of the probability amplitudes of a single-photon to take either of two possible paths" [9]. However, some physical contradictions arise: As $\hat{a}_{\mathrm{A}}^{\dagger}$ and $\hat{a}_{\mathrm{B}}$ operate simultaneously on mutually exclusive states, $|1\rangle_{\mathrm{A}}|0\rangle_{\mathrm{B}}$ and $|0\rangle_{\mathrm{A}}|1\rangle_{\mathrm{B}}$, the operator $\hat{a}_{\mathrm{B}}$, acting to the right, absorbs a photon, while a second photon needs to be absorbed by $\hat{a}_{\mathrm{A}}^{\dagger}$ as it acts to the left. Therefore, the two photons are associated with the mixed state of the ensemble of measurements, arriving at different times.

\section{The deficiencies of global quantum states}

Any probability amplitude of a quantum event should be evaluated from wavefunctions that reflect the physical reality. Discarding temporal information - which becomes a lack of information - about the propagation pathway of a single photon does not create a physical effect; it can only mask or obscure the existence of physical interactions.

The quantum Rayleigh spontaneous emission (QRSE) in a dielectric medium replaces entangled photons with independent ones [6-7]. A single photon cannot propagate in a straight line because of the QRSE. Only a group of monochromatic photons propagating together can maintain their line of propagation and properties because the absorbed photon may be recaptured through stimulated emission by the other photons in the group. A beam splitter does not conserve the number of photons passing through it because of the QRSE. For example, three photons entering through one input port of the BS are split at the dividing dielectric interface with two photons being reflected and one transmitted to the output ports of the BS. The latter photon will most likely be scattered randomly by QRSE and will not emerge from the second output port. As a result, the Hanbury Brown \& Twiss (HBT) measurement will indicate no coincidence for zerotime delay even though more than one photon were initially impinging onto the BS.

Equally, each photon carries an intrinsic optical field [6-8] which is distinct from the probability amplitude of possible pathways, and, at least, two photons need to be detected simultaneously by the same photodetector for an interference pattern to appear.

\section{The pure quantum state of individual measurements}

In the case of an HBT measurement with a stream of single photons launched into one of two inputs $a$ or $b$ of a lossless cubic prism BS, the outgoing annihilation operators from ports $c$ and $d$ are conventionally given by [10]: $\hat{a}_{c}=r \hat{a}_{a}$ and $\hat{a}_{d}=t \hat{a}_{a}$, or $\hat{a}_{c}=t \hat{a}_{b}$ and $\hat{a}_{d}=r \hat{a}_{b}$, with the reflection and transmission coefficients $|r|=|t|=\sqrt{ } 0.5$ and a relative phase of $\varphi_{r}-\varphi_{t}$ $= \pm \pi / 2$. With an input state of $|\Psi\rangle_{i n}=|1\rangle_{a}|0\rangle_{b}$, the ensemble probability $P$ of each HBT detector recording a single photon is [10]: $P={ }_{i n}\left\langle\Psi\left|\left(\hat{a}_{j}^{\dagger} \hat{a}_{j}\right)\right| \Psi\right\rangle_{i n}=|r|^{2}=|t|^{2}=0.5$, where $j=c$ or $d$. If the two output states are combined to interfere at one photodetector, then the joint detection probability is given by: $P_{\text {joint }}={ }_{i n}\left\langle\Psi\left|\left(\hat{a}_{c}^{\dagger}+\hat{a}_{d}^{\dagger}\right)\left(\hat{a}_{c}+\hat{a}_{d}\right)\right| \Psi\right\rangle_{i n}=|r+t|^{2}$ and the interference term is $2|r||t| \cos \left(\varphi_{r}-\varphi_{t}\right)=0$. The relative phase condition is arbitrarily chosen - without any physical mechanism being identified - in order to comply with the quantum interference postulate of adding the complex probability amplitudes for alternative quantum trajectories. If the BS is replaced with a symmetric optical waveguide Y-junction, then physically there would be no phase difference and the probability would exceed unity.

Another type of operator transformation by the BS has been suggested [11-12] with an output

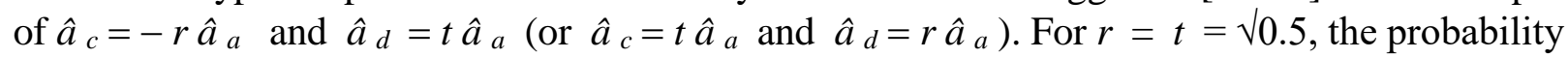


of one photon detection over the ensemble becomes for the first option $P_{\text {joint }}=0$, which is physically impossible.

Probabilities of a quantum event, such as photo-detection of a photon, are determined from a large ensemble of measurements carried out on identically prepared systems. Photonic ensembles for an HBT configuration will be described by a time-independent mixed state $|\Psi\rangle_{\text {en }}=$ $\sqrt{ } p_{\alpha}|1\rangle_{\mathrm{A}}|0\rangle_{\mathrm{B}}+\sqrt{ } p_{\beta}|0\rangle_{\mathrm{A}}|1\rangle_{\mathrm{B}}$ where the two ensemble distribution probabilities satisfy: $p_{\alpha}+p_{\beta}=1$. For this state, the interference term may be non-zero [3].

By contrast, a single HBT measurement will be represented by a time-dependent pure quantum state $|\Psi(t)\rangle_{m}==\alpha(t)|1\rangle_{\mathrm{A}}|0\rangle_{\mathrm{B}}+\beta(t)|0\rangle_{\mathrm{A}}|1\rangle_{\mathrm{B}}$ with $|\alpha(t)|^{2}+|\beta(t)|^{2}=1$, and the two mutually exclusive options, for one measurement, are: $|\alpha(t)|=1$ and $|\beta(t)|=0$, or $|\alpha(t)|=0$ and $|\beta(t)|=1$. Each HBT measurement indicates, at any given time, the existence of a photon in one of two branches and the absence of a joint interference term. These inconsistencies can be eliminated with a field-based approach, as the pure state describes the physical reality with a temporally and spatially localized single measurement of a photonic beam front.

\section{The intrinsic optical field of photons from the dynamic and coherent number states}

We can apply the Fresnel formulas of reflection and transmission if a wave function or state vector can be identified for the instantaneous optical field properties of a photonic wavefront, i.e. its amplitude in terms of the number $N$ of photons and its phase $\varphi$. Such a wavefunction is rather simple [6-8] taking the form

$\left.\left|\Psi_{\mathrm{n}}(t)\right\rangle=c_{\mathrm{n}}(t)|\mathrm{n}\rangle+c_{\mathrm{n}-1}(t)|\mathrm{n}-1\rangle\right)$ with $\left|c_{\mathrm{n}}\right|^{2}+\left|c_{\mathrm{n}-1}\right|^{2}=1$

and delivering a complex $c$ - number for the optical field amplitude and a real number $N$ for the photons carried by the wavefront [6-8]

$\left\langle\Psi_{\mathrm{n}}|\hat{a}| \Psi_{\mathrm{n}}\right\rangle=0.5 N^{1 / 2} \exp (-i \varphi)$

$0.5\left\langle\Psi_{\mathrm{n}}\left|\left(\hat{a} \hat{a}^{\dagger}+\hat{a}^{\dagger} \hat{a}\right)\right| \Psi_{\mathrm{n}}\right\rangle=N$

The wavefunction $\left|\Psi_{\mathrm{n}}\right\rangle$ will be referred to as the dynamic and coherent number state.

In the case of any number of photons, the role of the wavefunction is to deliver the required properties of the instantaneous number of photons, the corresponding amplitude and phase quadratures.

Based on the formalism presented in [13] the magnitude of the Poynting vector, i.e. the flux of energy $\mathscr{E}$ (or number of photons /s) carried by an optical wavefront of frequency $\omega$ and crossing a plane surface at position $z$ is given in terms of the electromagnetic field magnitudes $E$ and $B$, or corresponding operators, by the equalities:

$$
\mathscr{E}=\omega \varepsilon E^{2}+c^{2} \omega B^{2}=0.5 \hbar \cdot \omega\left(a a^{*}+a^{*} a\right)
$$

with $a=(\varepsilon / \hbar)^{1 / 2}(E+i c B)$ and its complex conjugate $a^{*}$. From this relation one defines the annihilation and creation operators as:

$$
\hat{a}=(\varepsilon / \hbar)^{1 / 2}(\hat{E}+i c \hat{B}) \quad \text { and } \quad \hat{a}^{\dagger}=(\varepsilon / \hbar)^{1 / 2}(\hat{E}-i c \hat{B})
$$

with $\varepsilon$ and $\hbar$ indicating the permittivity of the medium and the reduced Planck constant, respectively. The free-space Hamiltonian $\widehat{H}_{f}$ is explicitly written as: $\widehat{H}_{f}=\hbar \omega \widehat{N}_{c}$ with 
$\widehat{N}_{c}=0.5\left(\hat{a}^{\dagger} \hat{a}+\hat{a} \hat{a}^{\dagger}\right)$ the complete number operator and its eigenstates are the number states $|\mathrm{n}\rangle$ and $|\mathrm{n}+1\rangle$.

The correct expectation values can also be found for the quadrature operators [14], i.e.,

$$
\begin{aligned}
& \hat{C}=\widehat{a} \widehat{N}^{-1 / 2}+\widehat{N}^{-1 / 2} \hat{a}^{\dagger} \text { and } \hat{S}=i\left(\widehat{a} \widehat{N}^{-1 / 2}-\widehat{N}^{-1 / 2} \hat{a}^{\dagger}\right): \\
& \left\langle\Psi_{\mathrm{n}}\left|\hat{C}_{j}\right| \Psi_{\mathrm{n}}\right\rangle=\cos \left(\omega t+\varphi_{j}\right) \\
& \left\langle\Psi_{\mathrm{n}}\left|\hat{S}_{j}\right| \Psi_{\mathrm{n}}\right\rangle=\sin \left(\omega t+\varphi_{j}\right)
\end{aligned}
$$

These relations will prove useful in deriving the photon-coupling equations brought about by the quantum Rayleigh interactions [6-8].

We can calculate the intrinsic longitudinal field profile of a group of photons, or its coherence length, by using the wave function $\left|\Psi_{n}\right\rangle$ from eq. (1). Two equations can be identified for the expectation values of $\hat{a}$, leading to [6-8]:

$$
\left\langle\Psi_{\mathrm{n}}|\hat{a}| \Psi_{\mathrm{n}}\right\rangle=b\langle\hat{E}\rangle+i s\langle\hat{B}\rangle \quad \text { and } \quad\left\langle\Psi_{\mathrm{n}}|\hat{a}| \Psi_{\mathrm{n}}\right\rangle=q e^{-i \omega t}
$$

where $q=0.5 \mathrm{n}^{1 / 2}, b=(\varepsilon / \hbar)^{1 / 2}$ and, $s=(\varepsilon c / \hbar)^{1 / 2}$. We point out that both quadratures of the field are represented in the phasor notation of eq. (5). Recalling the relations [13] between the vector potential $\boldsymbol{A}(z, t)$ and the fields as: $\boldsymbol{E}=-\partial \boldsymbol{A} / \partial t$ and $\boldsymbol{B}=\nabla \times \boldsymbol{A}$ in the Cartesian frame of coordinates $(x, y, z)$, one solves the differential equation from equating equations (5) for the vector potential to obtain the longitudinal optical field profile $g_{f}$ of one photon of wavelength $\lambda$, crossing point $z_{\mathrm{o}}$, as

$g_{f}(\mathrm{z}=c t)=\exp \left(-2 \pi\left|z-z_{\mathrm{o}}\right| / \lambda\right)$

which has the form of a Wigner spectral component $S(\omega, t)$, that is, a time-varying spectral component [3], as opposed to a time-constant amplitude and phase of a Fourier spectrum. The transversality condition [13] for a radiation field $\langle\hat{E}\rangle \propto\left\langle\hat{a}+\hat{a}^{\dagger}\right\rangle$ and the dielectric constant $\varepsilon$, is: $\nabla \cdot(\varepsilon \boldsymbol{E})=0$. In cylindrical coordinates, the differential equation and its solution in the plane perpendicular to the wavevector of propagation are:

$\partial(r \varepsilon \boldsymbol{E}) / \partial r=0$ and $\boldsymbol{E}(r)=\boldsymbol{E}_{\mathrm{o}}\left(r_{\mathrm{o}} / \varepsilon r\right)$

This lateral distribution of the photonic field may explain the two-slit Young interference, as the broad lateral field splits at the slits and recombines behind them.

\section{Distorted outputs of single-photon sources}

It is pointed out in [15] that a quantum dot "emits a cascade of photons and a single photon is obtained only through spectral filtering of one emission line". High-finesse optical cavities incorporated in an HBT measurement setup distort the temporally regular sequence of single photons because of multiple internal reflections. The emerging stream may contain groups of a few overlapping photons, e.g. five, which may be unevenly split by a BS and reduced in number through QRSE so as to generate no coincidence for a zero delay-time.

A quantum dot (QD) placed in a high finesse micro-cavity of a few wavelengths and excited with a picosecond pulse, can emit a photon spontaneously and be re-excited within the duration of the same pulse. If the photon was reflected towards the QD, stimulated emission may occur 
due to the small dimensions of the micro-cavity. This will result in two, or more, photons leaving the emitter simultaneously as well as a reduced lifetime of the excited state of the QD, manifesting itself as a higher decay rate overshadowing the Purcell effect.

"Resonators with small mode volume and high-quality factors ( $Q$-factors) enhance the lightmatter coupling." [1]. However, a high $Q$-factor indicates a build-up of energy inside the cavity, i.e., a large number of photons do not leave the cavity after being emitted and reaching the output facet for the first time. The early photons emitted by a QD embedded in a resonant cavity of a dielectric structure of distributed Bragg reflectors (DBRs), may be reflected towards the excited quantum dot and be amplified, thereby giving rise to a time-varying spectral distribution which emerges from the "single-photon source" in the form of discrete pulses.

\section{Conclusions}

The probability amplitude approach to quantum interference leads to physical contradictions which are eliminated by using the intrinsic field of photons. Additionally, the statistics of a stream of single photons is distorted by an interferometric filter as multiple internal reflections occur randomly, and the relative time delays of photons vary.

\section{References}

1. R. Trivedi, K. A. Fischer, J. Vuckovic, and K. Müller, "Generation of Non-Classical Light Using Semiconductor Quantum Dots”, Adv. Quantum Technol., 3, 1900007, (2020).

2. J. C. Loredo, C. Antón, B. Reznychenko, P. Hilaire, A. Harouri, C. Millet, H. Ollivier, N. Somaschi, L. De Santis, A. Lemaître, I. Sagnes, L. Lanco, A. Auffèves, O. Krebs \& P. Senellart," Generation of non-classical light in a photon-number superposition", Nat. Photonics, 13, 803-808, (2019).

3. L. Cohen, "Time-frequency distributions-a review", 1989, Proc. IEEE, 77, 941-981.

4. D. Marcuse, Principles of Quantum Electronics, 1980, Academic Press.

5. W. H. Louisell, Quantum Statistical Properties of Radiation, 1973, John Wiley \& Sons.

6. A. Vatarescu, "The Scattering and Disappearance of Entangled Photons in a Homogeneous Dielectric Medium," Rochester Conference on Coherence and Quantum Optics (CQO-11), doi.org/10.1364/CQO.2019.M5A.19.

7. A. Vatarescu, The Physical Reality of Applied Quantum Optics, 2021 Cambridge Scholars Publishing.

8. A. Vatarescu, "Instantaneous Quantum Description of Photonic Wavefronts for PhaseSensitive Amplification," Frontiers in Optics/Laser Science Conference (FiO/LS), paper JW4A.109, Washington, Sept. 2018.

9. D. F. Walls, "A simple field theoretic description of photon interference," Am. J. Phys., 45, 952 - 956, (1977).

10. C. Garrison and R.Y. Chiao, Quantum Optics, Oxford University Press, 2008.

11. A. Kuhn \& D. Ljunggren," Cavity-based single-photon sources," Contemp. Phys., 51 (4), 289-313 (2010).

12. A. Reiserer and G. Rempe, "Cavity-based quantum networks with single atoms and optical photons," Rev. Mod. Phys., 87, No. 4, 1379 - 1418, 2015.

13. R. J. Glauber and M. Lewenstein, "Quantum optics of dielectric media," Phys. Rev. A, 43, 467- 491, (1991).

14. P. Carruthers and M. M. Nieto, "Phase and Angle Variables in Quantum Mechanics", Rev. Mod. Phys., 40, 411-440, (1968).

15. P. Senellart, G. Solomon, and A. White, "High-performance semiconductor quantum-dot single-photon sources," Nature Nanotech., 12, 1026- 1039, (2017). 